\title{
A Nonlinear Analytical Redundancy Method for Sensor Fault Diagnosis in an Automotive Application
}

\author{
Huanyi Shui ${ }^{1}$, Shiming Duan ${ }^{2}$, Chaitanya Sankavaram ${ }^{3}$, and ${\mathrm{Jun} \mathrm{Ni}^{4}}^{4}$ \\ ${ }^{1,4}$ University of Michigan, Ann Arbor, Michigan, 48105, USA \\ huanyis@umich.edu \\ junni@umich.edu \\ ${ }^{2,3}$ Vehicle System Research Lab, General Motors Global R\&D, Warren, Michigan, 48092, USA \\ shiming.duan@gm.com \\ chaitanya.sankavaram@gm.com
}

\begin{abstract}
Sensors have been widely implemented in vehicle systems for control, driving, and vehicle condition monitoring purposes. In a typical automotive vehicle, there are 60-100 sensors on board and is projected to reach 200 sensors per car. Those sensors provide rich information to ensure safe vehicle operation. However, like any dynamic systems, sensors are vulnerable to degrade or fail over time, which leads to the need of real-time sensor fault diagnosis. Analytical redundancy has been the key model-based approach for sensor fault diagnosis. However, existing analytical redundancy approaches are limited to linear systems, or some special cases of nonlinear systems. In this paper, the analytical redundancy approach is extended to nonlinear systems in general to ensure the accuracy of sensor measurements. Parity relations based on nonlinear observation matrix are formulated to characterize system dynamics and sensor measurements. Robust optimization is designed to identify the coefficient of parity relations that can tolerate certain level of measurement noise and model uncertainties. At last, sensor fault diagnosis in an air intake system is employed to demonstrate the effectiveness of the proposed method.
\end{abstract}

\section{INTRODUCTION}

The benefit of automated monitoring and control procedures advances the research development and usage of sensing techniques in engineering systems. For example, in a typical automotive vehicle, there are 60-100 sensors on board and is projected to reach 200 sensors per car for control, driving, and vehicle condition monitoring purposes. Those sensors

Huanyi Shui et al. This is an open-access article distributed under the terms of the Creative Commons Attribution 3.0 United States License, which permits unrestricted use, distribution, and reproduction in any medium, provided the original author and source are credited. play important roles in providing rich information to ensure safe vehicle operations. However, sensors might work in a severe and fast-changing environment with high pressure, high temperature or strong vibration. Like any dynamic systems, they are vulnerable to fail or degrade over time. Both abrupt (e.g., caused by corroded contacts) or incipient (e.g., caused by deteriorated sensing elements) can generate non-permitted deviations from characteristic properties in sensors and result in inaccurate measurements from monitored target variables (Isermann, 1984). Consequently, a sensor malfunction can lead to wrong control efforts, mislead vehicle diagnostics and prognostics, and affect vehicle performance.

To validate sensor measurements, both hardware redundancy and analytical redundancy approaches have been developed for sensor fault diagnosis. Hardware redundancy usually requires a high cost of extra sensor installation and maintenance, and often time is restricted by space and weight concerns. Analytical redundancy is more cost-effective and has been developed for many engineering applications. It employs mathematical models to describe the systems and generate residuals between the sensor measurements and model estimates for fault diagnosis. There have been many research studies of sensor fault diagnosis methods in linear systems, while for a nonlinear system, a common approach is to apply those methods after linearizing the nonlinear system, which often suffers from inaccuracy because of modeling errors. Therefore, sensor fault diagnosis in nonlinear systems still remains challenging.

To conquer the challenge, this research aims at developing a real-time sensor fault diagnosis method in general nonlinear systems by proposing a model-based nonlinear analytical redundancy approach. Nonlinear observation matrix is employed to derive input-output equations to describe system dynamics and sensor measurements. Robust optimization is 
designed to obtain best coefficients so that the generated residuals will be robust to noise and uncertainties, but sensitive to sensor failures.

In the following, the literature review of sensor fault diagnosis is shown in Section 2 and the problem formulation of nonlinear analytical redundancy is presented in Section 3. In Section 4, the proposed modeling method for parity residual generation in a nonlinear system is proposed. The robust optimization is employed to obtain model coefficients. A case study of sensor fault diagnosis in an air intake system is detailed in Section 5 and the conclusion is in Section 6.

\section{LITERATURE REVIEW OF SENSOR FAULT DIAGNOSIS}

A general solution to validate sensor measurements in a realtime environment is to add redundancy in the system. Hardware redundancy is the most intuitive approach that has been applied to many quality/safety-critical systems. It adds additional sensors to measure critical targets in a system and check the consistency among redundant sensors to detect if any sensor is faulty. However, additional sensors require extra cost, weight, and space. Even though the recent evolution of micro-technology has contributed to reducing the size and cost of sensors, the hardware redundancy approach is still not applicable in many industrial applications. Moreover, redundant sensors may fail or degrade in the same way of the primary sensor since they all work under the similar operating environment (Patton et al., 1989). At last, when multiple sensors fail, the hardware redundancy approach tends to be infeasible to detect sensor failures under majority voting scheme (Broen, 1974).

Analytical redundancy provides a promising solution that is independent of redundant sensors. Both qualitative and quantitative models have been developed to add analytical redundancies in systems to check the accuracy of sensor measurements. Qualitative models are mainly built based on qualitative and heuristic reasoning or causal relationship between observations and system performance. Quantitative models employ mathematical expressions to represent system dynamics and estimate sensor measurements under fault-free conditions. With a nominal model and real-time sensor measurements, residuals are generated to detect and isolate sensor failures using various methodologies. The successful deployment of analytical approaches highly relies on model accuracy, which is affected by different levels of measurement noise and model uncertainties under different operating conditions.

The state-of-the-art modeling methods to achieve analytical redundancy for sensor fault detection and isolation can be further categorized into three: model-based methods, knowledge-based expert systems and data-driven methods (Jiang, 2011). The model-based methods such as parity relations (Chow and Willsky, 1984), Luenberger observers and Kalman filtering, (Clark, 1978; Tesheng Hsiao and Tomizuka, 2005; Du and Mhaskar, 2014) and parameter estimators (Upadhyaya and Kerlin, 1987) can develop quantitative models to generate for sensor fault diagnosis. However, those methods require thorough knowledge of target system dynamics to formulate high fidelity models, which is often not applicable for complex systems. Knowledge-based expert systems (Betta et al., 1995; Kim, 1997) such as lookup table, fault tree and fuzzy logic based methods require comprehensive engineering domain knowledge of system behaviors under various normal conditions and faulty conditions. It has limited capability to handle dynamic systems especially during the initial development phase due to its rule-based mechanisms. Datadriven methods such as artificial neural networks (Mathioudakis and Romessis, 2004; Elnokity et al., 2012) and multivariate statistical methods (Negiz and Cinar, 1992; Dunia et al., 1996; Huang et al., 2000) are able to handle complex systems but require sufficient data to learn data patterns or trends to represent system performance, and usually lack physical insights. In real practice, those methods can be integrated to leverage their own advantages and disadvantages so that to obtain representative symptoms for diagnostics/prognostics. Nevertheless, it is usually preferable to start with model-based methods when physical knowledge is available.

In literature, the standard model-based analytical methods have been developed for linear systems (Chow and Willsky, 1984; Qin and Li, 2001; Li and Shah, 2002). However, many engineering systems are nonlinear. Nonlinearity does not obey superposition principles, and tends to introduce discontinuity and unpredictable output into systems. Those properties make the implementation of nonlinear analytical redundancy difficult. Many efforts have been made to linearize nonlinear systems in order to apply linear analytical redundancy methods (Nguang et al., 2007). However, nonlinear systems suffer considerably from linearization since it may introduce errors and reduce model accuracy. The performance of model-based analytical redundancy methods is sensitive to inconsistencies between nominal models and actual system behaviors. Therefore, those errors will affect the effectiveness of linear analytical redundancy methods for sensor fault diagnosis in nonlinear systems. Other researchers explored nonlinear analytical redundancy methods for some special cases of nonlinear systems whose nonlinear observation matrix can be formulated into a linear form in terms of inputs and outputs (Yu and Shields, 2001; Shumsky, 2008; Leuschen et al., 2005). However, those nonlinear analytical methods are only feasible for specific system types, therefore, lack generality. A general form of nonlinear analytical redundancy approach is still not available.

To fill the gap, this study will investigate a model-based analytical redundancy method for the sensor fault diagnosis problem in general nonlinear systems in which, both input 
and output equations are nonlinear functions of states and inputs. Following the idea of the linear analytical redundancy method that utilizes observation matrix to construct inputoutput relations to describe the relationships between system behaviors and sensor measurements, this study will employ nonlinear observation matrix that is derived from system dynamic equations in the control theory to build the inputoutput relations with a parity space approach. The number of available analytical redundancies that can be added for sensor fault diagnosis will be determined by the rank of the nonlinear observation matrix.

\section{Problem Formulation}

Consider a general nonlinear system with $N$ states, $Q$ measurable inputs and $M$ sensors:

$$
\begin{aligned}
& \dot{x}=f(x, u)+\varepsilon \\
& y=h(x, u)+\delta
\end{aligned}
$$

where $x \subseteq R^{N}$ is the state vector, $u \subseteq R^{Q}$ is the measurable input vector, $y \subseteq R^{M}$ is the sensor measurements, $\varepsilon$ is the system disturbance and $\delta$ is the measurement noise. Different from existing works, the system here is a general form, in which both the input equation and the output equation are nonlinear functions of states and inputs. Thus, the formulation of nonlinear analytical redundancy should be flexible to represent the dynamic behavior of the system by considering its dependence on both states and inputs.

A model-based analytical redundancy method exploits the null-space of the state space observation matrix to generate residuals for fault diagnosis. Those residuals contain the complete information from sensor data and actuator inputs to detect any deviations from the nominal behavior of sensors. Existing works for nonlinear systems only have addressed those system models that can be linearized (Nguang et al., 2007) or use a simplified nonlinear observation matrix to formulate a structure for residuals generation (Leuschen et al., 2005). Those approximations will introduce a considerable amount of model errors so that, for general nonlinear systems, they sometimes can hardly provide effective solutions for accurate sensor fault diagnosis.

In order to formulate effective analytical redundancy for general nonlinear systems, the notion of observability is employed. Observability is a fundamental measure in control theory, which reflects the possibility for estimating intermediate states based on input and output signals.

Definition 3.1: The system is locally observable at $x_{0}$ if there exists a neighborhood of $x_{0}$ such that every $x$ in that neighborhood other than $x_{0}$ is distinguishable from $x_{0}$. The mathematical expression for checking local observability is:

$$
\operatorname{Rank}\left(\nabla O\left(x_{0}, u^{*}\right)\right)=N
$$

where $N$ is the rank of $x$. For each output $y_{i},(i=1, \ldots, M)$

$$
\begin{gathered}
{\left[\begin{array}{c}
y_{i} \\
\dot{y}_{i} \\
\ddot{y}_{i} \\
\vdots
\end{array}\right]=\left[\begin{array}{c}
L_{f}^{0} \\
L_{f}^{1} \\
L_{f}^{2} \\
\vdots
\end{array}\right] h\left(x_{0}, u^{*}\right)=O\left(x_{0}, u^{*}\right)} \\
L_{f}^{k}=L_{f}\left(L_{f}^{k-1} h_{i}\right) \text { and } L_{f}^{0} h_{i}=h_{i} \\
L_{f}^{1} h_{i}=\frac{\partial h_{i}}{\partial x} f+\frac{\partial h_{i}}{\partial u} \frac{d u}{d t}
\end{gathered}
$$

where $O$ is a nonlinear observation matrix, and $L_{f}^{1} h_{i}$ is the Lie derivative of $h_{i}$ with respect to $f$. In nonlinear systems, nonlinear observability is only feasible in its local space that is close to $x_{0}$ and $u^{*}$. It also requires the system to be smooth so that the Lie derivative $L_{f}^{k} h_{i}$ exists. It is assumed that the major operating regime meets this requirement in this study.

From the definition, it is seen that the second term $\frac{\partial h_{i}}{\partial u} \frac{d u}{d t}$ on the right-hand side of Eq. (5) is the main difference between this work and others. Existing methods do not consider the situation that the output $y_{i}$ is related to inputs. As a result, the effect of this term is neglected. In this research, a complete form of analytical redundancy structure for general nonlinear systems is proposed.

Moreover, the locally observable property indicates that the observability in a nonlinear system is valid within a certain working region that is near the current states and inputs. In real practice, when various operating conditions exist, an operating regime with the same level of observability has to be identified, and the entire working space has to be partitioned according to different operational and environmental conditions. This paper assumes the operation conditions are pre-defined by engineering knowledge, while the automatic operating partition of space would be the future work. Moreover, this study will employ robust optimization method to identify the coefficients for local analytical redundancies in the pre-defined operating condition that around an operating point $\left(x_{0}\right.$ and $\left.u^{*}\right)$.

To sum up, this study focuses on the formulation of the nonlinear analytical redundancy in general nonlinear systems. A complete form of analytical redundancy structure is proposed for sensor fault diagnosis. A robust optimization is designed to identify the model coefficients so that the generated residuals are projected to a space where they are sensitive to sensor failures but robust to noise and uncertainties. The resulting residuals generated from the proposed analytical redundancies can be utilized to identify sensor failures or quantify its degradation status. The diagnostic capability of the proposed method in nonlinear systems is demonstrated and validated with data from an air intake system. 


\section{NONLINEAR ANALYTICAL REDUNDANCY IN GENERAL NONLINEAR SYSTEMS}

The model-based analytical redundancy method exploits the basic concept of observability, which contains key information of the system behaviors that can be inferred from the observation space. Also, a proper design of analytical redundancy is able to generate linearly independent residuals so that all detectable deviations from the system models can be accounted. In this section, the parity space method is employed to add analytical redundancies in the system. To address the sensor fault diagnosis problem in nonlinear systems, the traditional parity space model is extended from linear to nonlinear systems so that input-output equations can be formulated to generate residuals. Furthermore, robust optimization design is employed to obtain optimal coefficients for the parity space model so that parity residuals will be close to zero when only measurement noise and model uncertainty involve, while will have large magnitudes when sensor failures occur in the system.

\subsection{Parity Residual Generation}

The parity space captures key information of system dynamics from the observation space. As shown in Figure 1, parity relations characterize relationships among measurable inputs and sensor outputs so that a set of residuals can be obtained (Chow and Willsky, 1984). In addition, the linear independence property in parity space guarantees that every parity residual generated contains at least some information that has not been covered by other residuals. On the other hand, each observable deviation from the system model is covered by at least one of the parity residuals.

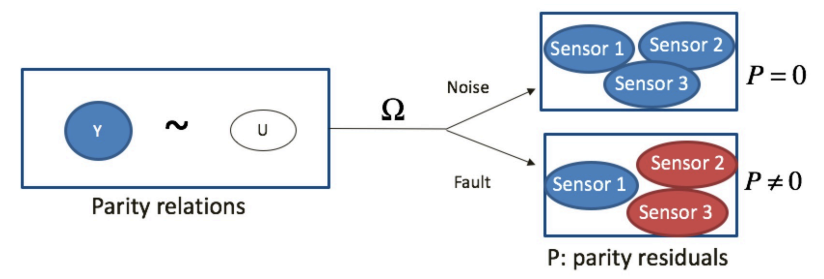

Figure 1. Sensor fault diagnosis with the parity space approach

The nonlinear observability imposes a prerequisite to construct the parity space that allows analytical redundancy for general nonlinear systems. In the following, based on Definition 3.1, the complete parity relations are derived, and the parity space is formed with

$$
\Omega^{\perp} O\left(x_{0}, u^{*}\right)=0
$$

where $\Omega$ is a vector of nonzero coefficients that transfers residuals from original space to parity (null) space.

A straightforward approach to formulate the input-output equations for analytical redundancies in general nonlinear systems is to follow the methods in linear systems, which formulate analytical redundancy structures with linear functions of parameters (Chow and Willsky, 1984). However, due to the nonlinear form of both states and inputs, it is difficult to directly adapt the linear analytical redundancy structure for the general nonlinear systems. Also, since the output $y$ depends on both states and inputs, it is also not applicable to simplify the nonlinear observation matrix, or to formulate the input-output equations as shown in (Leuschen et al., 2005).

Instead of manipulating the observability matrix, the nonlinear observation matrix $O$ is decomposed into two parts: one is with respect to $x$, denoted as $O_{N L}$ while the other one is with respect to $[u, \dot{u}, \ddot{u}, \cdots]$ denoted as $E U$. The observation matrix in Eq. (3) is revised as

$$
\begin{aligned}
& O\left(x_{0}, u^{*}\right) \\
& =\left[\begin{array}{c}
h_{j} \\
\frac{\partial L_{f}^{0} h_{j}}{\partial x} f \\
\frac{\partial L_{f}^{0} h_{j}}{\partial x} f \\
\vdots
\end{array}\right]_{x_{0}, u^{*}}+\left[\begin{array}{cccccc}
0 & 0 & 0 & 0 & 0 & \cdots \\
0 & \frac{\partial L_{f}^{0} h_{j}}{\partial u} & 0 & 0 & 0 & \cdots \\
0 & \frac{\partial L_{f}^{1} h_{j}}{\partial u} & \frac{\partial L_{f}^{1} h_{j}}{\partial \dot{u}} & 0 & 0 & \cdots \\
\vdots & \vdots & \vdots & \ddots & 0 & \vdots
\end{array}\right]_{x_{0}, u^{*}}\left[\begin{array}{c}
u \\
\dot{u} \\
\ddot{u} \\
\vdots
\end{array}\right] \\
& =O_{N L}+E U
\end{aligned}
$$

The first part $O_{N L}$ in Eq. (7) is used to determine $\Omega$ in Eq. (6) so that the parity (null) space can be formulated. According to the nonlinear observability of general nonlinear systems, the Eq. (6) is re-derived as:

$$
\Omega^{\perp} \nabla_{x} O_{N L}\left(x_{0}, u^{*}\right)=0
$$

where for the $j^{\text {th }}$ sensor

$$
\nabla_{x} O_{N L}\left(x_{0}, u^{*}\right)=\nabla_{x}\left[\begin{array}{c}
h_{j} \\
\frac{\partial L_{f}^{0} h_{j}}{\partial x} f \\
\vdots \\
\frac{\partial L_{f}^{m_{j}} h_{j}}{\partial x} f
\end{array}\right]_{x_{0}, u^{*}}
$$

The number of redundancies in this system is determined by the rank of each $\nabla O\left(x_{0}, u^{*}\right)$. For the $j^{\text {th }}$ sensor, the rank is determined as:

$$
m_{j}=\operatorname{rank}\left(\nabla_{x} O\left(x_{0}, u^{*}\right)\right)
$$

Here, the system can be either observable or unobservable. The number of independent analytical redundancies is denoted as:

$$
n-N=\sum_{j=1}^{M}\left(m_{j}+1\right)-N
$$


$n-N$ sets of independent $\Omega$ is determined to formulate the parity space i.e., the dimension of $\Omega$ is $\left(\sum_{j=1}^{M}\left(m_{j}+1\right)-\right.$ $\mathrm{N}) \times \sum_{j=1}^{M}\left(m_{j}+1\right)$.

The second part - EU in the Eq. (7) mainly represents the information of inputs, and is used to construct analytical redundancy structure in parity space. $E U$ is moved to the side of $y$ in Eq. (3) so that parity relations for the general nonlinear systems can be formulated as:

$$
P=\Omega^{\perp}\left\{\left[\begin{array}{c}
Y_{1} \\
Y_{2} \\
\vdots \\
Y_{M}
\end{array}\right]-\left[\begin{array}{c}
E_{1} \\
E_{2} \\
\vdots \\
E_{M}
\end{array}\right] U\right\}=0
$$

where $P$ is parity residuals, which has the dimension of $\sum_{j=1}^{M}\left(m_{j}+1\right) \times 1$ and ideally, will be nonzero only if a failure presents and the $E_{j}$ matrix is derived as

$$
E_{j}=\left[\begin{array}{ccccccc}
0 & 0 & 0 & 0 & 0 & \cdots & \cdots \\
0 & \frac{\partial L_{f}^{0} h_{j}}{\partial u} & 0 & 0 & 0 & \cdots & \cdots \\
0 & \frac{\partial L_{f}^{1} h_{j}}{\partial u} & \frac{\partial L_{f}^{1} h_{j}}{\partial \dot{u}} & 0 & 0 & \cdots & \cdots \\
\vdots & \vdots & \vdots & \ddots & \vdots & \vdots & \vdots \\
0 & \frac{\partial L_{f}^{m_{j}-1} h_{j}}{\partial u} & \frac{\partial L_{f}^{m_{j}-1} h_{j}}{\partial \dot{u}} & \cdots & \frac{\partial L_{f}^{m_{j}^{-1}} h_{j}}{\partial u^{m_{j}-1}} & 0 & \cdots
\end{array}\right]
$$

where $\mathrm{E}=\left[\begin{array}{llll}E_{1} & E_{2} & \cdots & E_{M}\end{array}\right]$ is a $\sum_{j=1}^{M}\left(m_{j}+1\right) \times \max \left(m_{j}\right) Q$ matrix when the rank of $j^{\text {th }}$ sensor is $m_{j} . \mathrm{U}=$ $\left[\begin{array}{llll}U_{1} & U_{2} & \cdots & U_{M}\end{array}\right]$ is a matrix with dimensionmax $\left(m_{j}\right) Q \times 1$ and $U^{q}=\left[\begin{array}{llll}u_{q} & \frac{d u_{q}}{d t} & \cdots & \frac{d^{\max \left(m_{j}\right)} u_{q}}{d t^{\max \left(m_{j}\right)}}\end{array}\right]^{T}$ for the $q^{t h}$ input.

Essentially, the parity relations can be viewed as a weighted combination of sensor outputs and actuator inputs. The structure of a parity relation defines what should be included, while the coefficients of the parity relation determine the weights.

Table 1 lists the comparison of parity residuals generation in linear systems and general nonlinear systems. It shows that the notion of nonlinear analytical redundancy with the parity relation method for general nonlinear systems is analogous to the notion of linear analytical redundancy.

Table 1: Parity relations in linear systems vs. general nonlinear systems

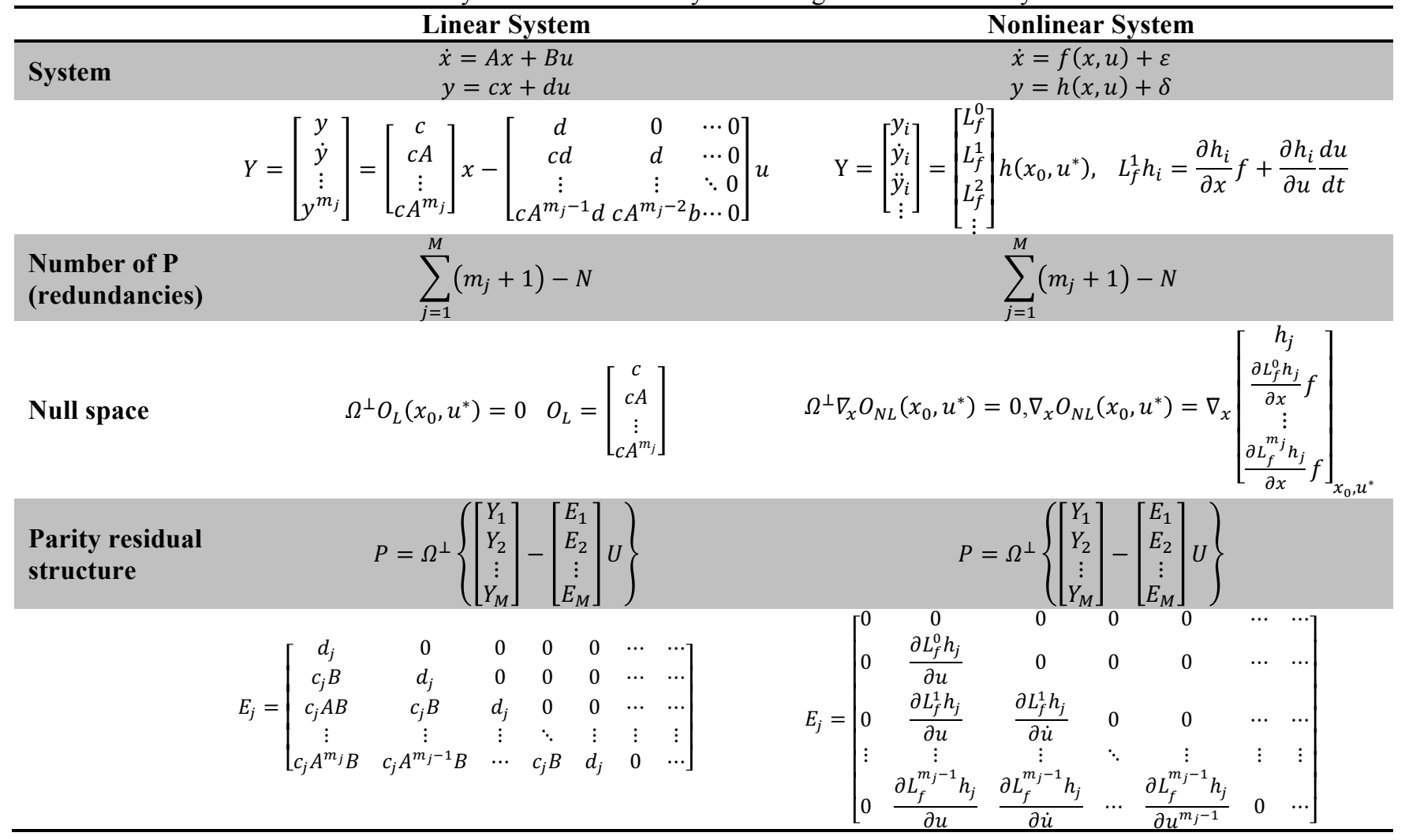




\subsection{Parity structure and coefficient design}

Given the general formulation of parity relations in the previous section, one is faced with the problem of finding the best structure and coefficients so that parity residuals are projected to a space where they are robust to noise but sensitive to sensor failures. The local property of nonlinear observability may lead to different parity structures under different operating conditions. Moreover, the statistical characteristics of parity residuals may be affected significantly from one operating condition to another because of different levels of measurement noise and modeling uncertainties. In order to provide accurate sensor fault diagnosis, the working space needs to be partitioned to determine local parity structures and coefficients. The automatic working space partition method will be the future work. While in this section, it is assumed that operating conditions are well defined. In many applications, measurement noise and model uncertainties in each operating condition will lead to the difficulty of selecting $\Omega$ such that $P$ $=0$. Therefore, the robust optimization is designed to find the best choice of coefficients that can make the candidate parity relations close to zero under no-failure conditions, and the resulting parity residuals can provide significant failure signatures to indicate anomalies or sensor failures.

The $\Omega$ is determined so that the value of $P$ is minimized in the existence of measurement noise and model uncertainties. The optimization formulation is shown as below:

$$
\begin{aligned}
& J^{*}=\min _{\Omega} \max _{\varepsilon, \delta} P^{2} \\
& P^{2}=\left\{\Omega^{\perp}(Y-E U)\right\}^{2}
\end{aligned}
$$

Here, the quantity of $\max _{\varepsilon, \delta} P^{2}$ is the worst case effect of noise and model uncertainty on the parity relations. A conservative choice is attempted to find the parity coefficients by minimizing the worst case.

However, it has a trivial solution that all coefficients are zero. To provide a meaningful solution, the coefficients $\Omega$ are constrained to have unit magnitude. Moreover, based on the mechanism of parity relation, another constraint is that an optimal set of $\Omega$ projects the observation matrix to a null space. Therefore, the complete formulation of a robust optimization design is shown as following:

$$
\begin{aligned}
& \min _{\Omega} \max _{\varepsilon, \delta} P^{2}=\left\{\Omega^{\perp}(Y-E U)\right\}^{2} \\
& \text { s.t. } \Omega^{\perp} \Omega=1 \\
& \quad \Omega^{\perp} \nabla O_{N L}\left(x_{0}, u^{*}\right)=0
\end{aligned}
$$

Based on the number of analytical redundancies, n-M sets of independent $\Omega$ will be selected.

Notably, the quantity of $\max _{\varepsilon, \delta} P^{2}$ is dependent on state $\mathrm{x}$ and input $u$, which indicates that the coefficients should be computed at each time step when state $\mathrm{x}$ and input $\mathrm{u}$ are changing over time. However, it is not desirable to obtain new coefficient all the time. A more applicable approach is to schedule the coefficients based on the operating conditions since a set of coefficients is usually effective for a range of $\mathrm{x}$ and $u$. This indicates that when the state and the inputs are varying at a certain range, the corresponding coefficients are likely to perform closely to the optimum. In this case, appropriate coefficients will be learnt for each operating condition which is characterized by some nominal state $\mathrm{x}$ and input $u$ during the training process and can be retrieved for use at corresponding operating conditions.

\section{A Case Study}

The air intake system is a safety-critical system in automotive vehicles. It consists of three main parts: air filter, throttle body, and intake manifold. As shown in Figure 2, there are three critical sensors in this system - manifold absolute pressure (MAP) sensor, mass air flow (MAF) sensor and throttle position sensor (TPS). Right after the air filter, a MAF sensor measures the air flow rate entering the system. The throttle body controls the amount of air flowing into the engine, and a throttle position sensor provides feedback on the throttle plate position. A MAP sensor captures the intake manifold pressure information in the intake manifold whose main function is to distribute combustion mixture to each intake port in the cylinder heads evenly.

This case study focuses on sensor fault diagnosis for both MAF and MAP sensors in an air intake system, considering the throttle position as a controllable input. It is assumed that input signals are known, and no faults are present in actuators.

Due to the confidentiality required by our research sponsor, details of the system model are not listed. In the following, a general nonlinear system will be formulated based on the system dynamics of the air intake system. The diagnostic capability of the designed analytical redundancies will be demonstrated with normal data from a testbed and abnormal data by introducing simulated sensor faults into the normal data.

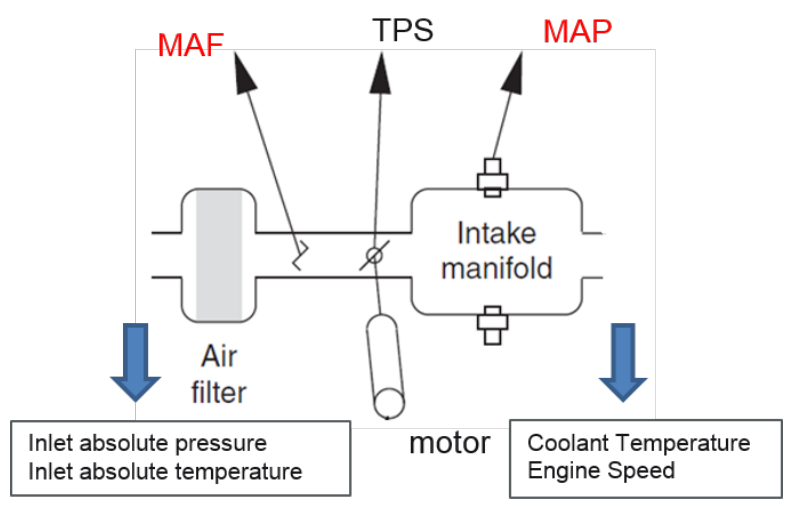

Figure 2. The layout of an air intake system 


\subsection{Parity structure and coefficient design}

To validate the proposed method, a state space model for the air intake system is constructed as a general nonlinear system with one state, one actuator input, and two sensor measurements $\mathrm{A}$ and $\mathrm{B}$.

$$
\begin{gathered}
\dot{x}=f(x, u)+\varepsilon \\
y_{A}=h_{1}(x, u)+\delta_{1} \\
y_{B}=h_{2}(x, u)+\delta_{2}
\end{gathered}
$$

By examining the observation matrix of $y_{A}$ and $y_{B}$, the rank of each sensor measurement is one so that in total, three analytical redundancies exist in this system. The model estimations of $y_{A}$ and $y_{B}$ are compared with real sensor measurements. Figure 3 shows that the model accuracy differs under different operating conditions. Under operating condition 1, the estimation error of sensor A is much smaller than the error generated under operating condition 2 . The main reason for this difference is model uncertainty and sensor measurement noise induced by different operating conditions. Here, the entire working space is partitioned into two operating regimes according to the speed of the production system.
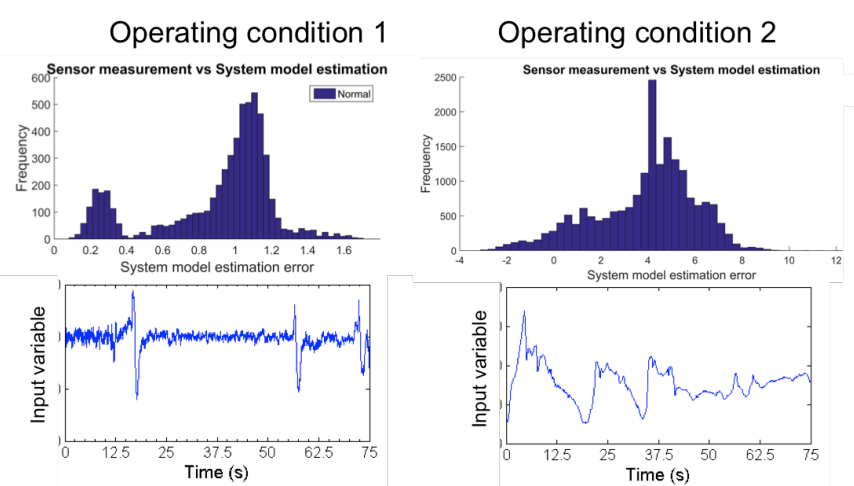

Figure 3. State space model estimation error of sensor A under different operating conditions

Based on the observation matrix of the state space model in Eq. (16), the parity structure is formulated as:

$$
\mathrm{P}=\Omega^{\perp}\left\{\left[\begin{array}{l}
y_{A} \\
\dot{y}_{A} \\
y_{B} \\
\dot{y}_{B}
\end{array}\right]-\left[\begin{array}{c}
0 \\
\frac{\partial h_{1}}{\partial u} \\
0 \\
\frac{\partial h_{2}}{\partial u}
\end{array}\right] \dot{u}\right\}
$$

and

$$
O_{N L}\left(x_{0}, u^{*}\right)=\left[\begin{array}{c}
h_{1} \\
\frac{\partial h_{1}}{\partial x} f \\
h_{2} \\
\frac{\partial h_{2}}{\partial x} f
\end{array}\right]
$$

Based on the rank of the observation matrix of each sensor, there are three independent analytical redundancies available so that three sets of parity coefficients $\Omega^{1}, \Omega^{2}$, and $\Omega^{3}$, are determined by the robust optimization design in Eq. (15) with nominal data from operating condition 1, shown in Table 2.

Table 2: Selected parity coefficients under operating condition 1

\begin{tabular}{ccccc}
\hline $\boldsymbol{\Omega}$ & $y_{A}$ & $\dot{y}_{A}$ & $y_{B}$ & $\dot{y}_{B}$ \\
\hline $\boldsymbol{\Omega}^{\mathbf{1}}$ & -0.2125 & -0.0081 & 0.9771 & 0 \\
$\boldsymbol{\Omega}^{\mathbf{2}}$ & 0 & 0 & 0.9998 & -0.0125 \\
$\boldsymbol{\Omega}^{\mathbf{3}}$ & 0.9993 & 0.0369 & 0 & 0 \\
\hline
\end{tabular}

\subsection{Parity structure and coefficient design}

In order to demonstrate the effectiveness of the designed parity relations based on the proposed method for sensor fault detection in the air intake system, different levels of sensor faults are introduced into sensors A and B, respectively. On the left side of Figure 3, different amounts of offsets are added to nominal sensor A measurements, while on the right side, different gains are added to sensor B. Those faults are labeled as N05, N15, and P05, P15. The letters N and P present negative and positive offsets/gains that are added to the sensor measurements. The number quantifies the amount of offsets/gains that are introduced into the sensor measurements. A larger number indicates more severe faults/degradation.

Figure 4 shows that when offsets are added to sensor A, parity residuals governed by $\Omega^{1}$, and $\Omega^{3}$ show deviations from their nominal condition, while when gains are added to sensor B, deviations only appear in those parity residuals with $\Omega^{1}$ and $\Omega^{2}$. It indicates that under operating condition 1 , the parity residuals with $\Omega^{1}$ contain both information from sensor A and $\mathrm{B}$, while the parity residuals with $\Omega^{3}$ and $\Omega^{2}$ only contain the individual information for sensors A and $\mathrm{B}$, respectively. Moreover, when more severe faults are introduced into the sensor measurements, larger deviations can be found in the parity residuals from nominal values. Therefore, those parity residuals can provide effective information for sensor fault detection with the false alarm rate $1.06 \%$ under operating condition 1 . 

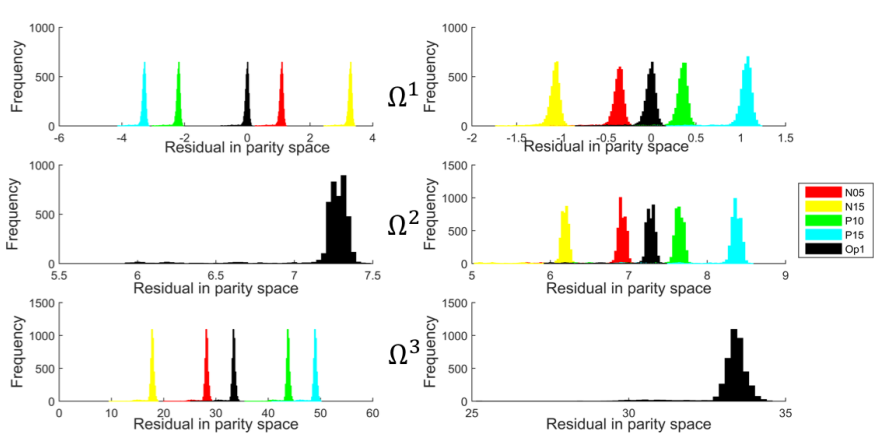

Figure 4. Distribution of parity residuals under different levels of sensor faults (Left: offsets added to sensor A;

Right: gains added in sensor B)

However, when the parity coefficients determined by normal data from operating condition 1 are used to generate parity residuals with nominal data from operating condition 2 , the false alarm rate is increased to $91.51 \%$. As shown in Figure 4 , the deviations will lead us to misjudge system sensor measurement noise/model uncertainties as a sensor fault. Such high false alarm rate is because the performance of sensor fault detection via parity space is only feasible around a certain range of inputs and state due to the local observability property in nonlinear systems, and is sensitive to the inevitable uncertainty in the knowledge of system dynamics and measurement noise under different operating conditions.
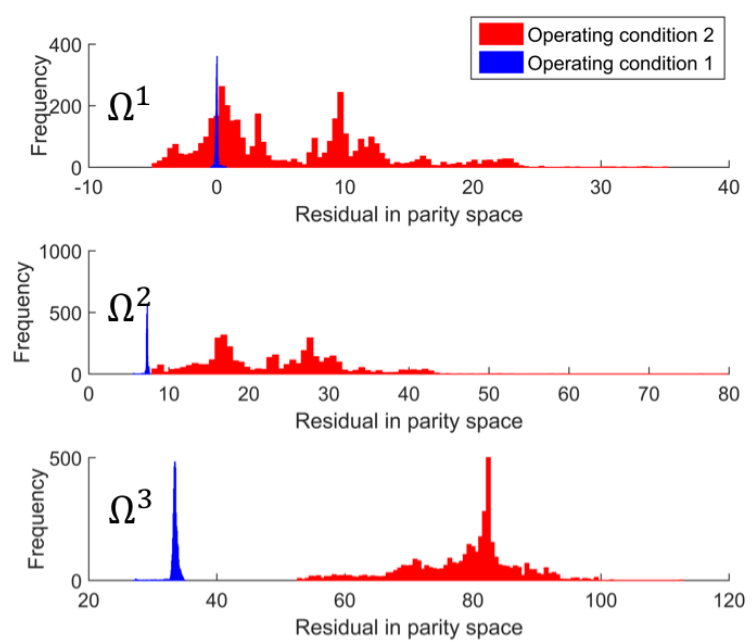

Figure 5. Distribution of parity residuals under different operating conditions

\section{CONCLUSION}

This research extends the model-based analytical redundancy from linear systems to general nonlinear systems, which fills the gap of analytical redundancy approaches for sensor fault diagnosis in general nonlinear systems, where input and output equations are nonlinear functions of both state variables and input variables. The notion of parity relations is derived based on the nonlinear observation matrix from system dynamic equations, which construct a parity space for sensor fault diagnosis in nonlinear systems. A robust optimization problem is formulated to find the best coefficient for the parity relations against the sensor measurement noise and model uncertainty under a certain operating condition. The case study validates the proposed method with data from an air intake system. The result shows that the proposed method is capable of identifying sensor degradation with different severity in a nonlinear system under the operating range it designed. It demonstrates the necessity of autonomous working space partition to construct local parity relations for sensor fault diagnosis, which could be a future work.

\section{ACKNOWLEDGEMENT}

The author would like to thank the technical and financial support from the research team in Vehicle Health Management at the General Motor.

\section{REFERENCES}

Betta, G, D’Apuzzo, M, Pietrosanta, A. (1995). A knowledge-based approach to instrument fault detection and isolation. IEEE Trans. Instrum. Meas. 44: 1009-1016.

Broen, R. (1974). A nonlinear voter-estimator for redundant systems. In: 1974 IEEE Conference on Decision and Control including the 13th Symposium on Adaptive Processes. IEEE, p 743-748.

Chow, E, Willsky, A. (1984). Analytical redundancy and the design of robust failure detection systems. IEEE Trans. Automat. Contr. 29: 603-614.

Clark, RN. (1978). Instrument Fault Detection. IEEE Trans. Aerosp. Electron. Syst. AES-14: 456-465.

Du, M, Mhaskar, P. (2014). Isolation and handling of sensor faults in nonlinear systems. Automatica 50: 10661074.

Dunia, R, Qin, SJ, Edgar, TF, McAvoy, TJ. (1996). Identification of faulty sensors using principal component analysis. AIChE J. 42: 2797-2812.

Elnokity, O, Mahmoud, II, Refai, MK, Farahat, HM. (2012). ANN based Sensor Faults Detection, Isolation, and Reading Estimates - SFDIRE: Applied in a nuclear process. Ann. Nucl. Energy 49: 131-142.

Huang, Y, Gertler, J, McAvoy, TJ. (2000). Sensor and actuator fault isolation by structured partial PCA with nonlinear extensions. J. Process Control 10: 459-469.

Isermann, R. (1984). Process fault detection based on modeling and estimation methods-A survey. Automatica 20: 387-404.

Jiang, L. (2011). Sensor Fault Detection and Isolation Using System Dynamics Identification Techniques.

Kim, Y. (1997). A framework for an on-line diagnostic expert system with intelligent sensor validation. KSME Int. J. 11: 10-19.

Leuschen, ML, Walker, ID, Cavallaro, JR. (2005). Fault 
residual generation via nonlinear analytical redundancy. IEEE Trans. Control Syst. Technol. 13: $452-458$.

Li, W, Shah, S. (2002). Structured residual vector-based approach to sensor fault detection and isolation. J. Process Control 12: 429-443.

Mathioudakis, K, Romessis, C. (2004). Probabilistic neural networks for validation of on-board jet engine data. Proc. Inst. Mech. Eng. Part G J. Aerosp. Eng. 218: 5972.

Negiz, A, Cinar, A. (1992). On the detection of multiple sensor abnormalities in multivariate processes. In: American Control Conference, 1992. Chicago, IL, USA: IEEE, p 2364-2368.

Nguang, SK, Zhang, P, Ding, S. (2007). Parity Relation Based Fault Estimation for Nonlinear Systems: An LMI Approach1. In: Fault Detection, Supervision and Safety of Technical Processes 2006. Elsevier, p 366371.

Patton, R, Clark, R, Frank, PM. (1989). Fault diagnosis in dynamic systems: theory and application. New York, USA: Prentice Hall.

Qin, SJ, Li, W. (2001). Detection and identification of faulty sensors in dynamic processes. AIChE J. 47: 15811593.

Shumsky, A. (2008). Robust Analytical Redundancy Relations for Fault Diagnosis In Nonlinear Systems. Asian J. Control 4: 159-170.

Tesheng Hsiao, Tomizuka, M. (2005). Sensor fault detection in vehicle lateral control systems via switching kalman filtering. In: Proceedings of the 2005, American Control Conference, 2005. IEEE, p 5009-5014.

Upadhyaya, BR, Kerlin, TW. (1987). Estimation of response time characteristics of platinum resistance thermometers by the noise analysis technique. ISA Trans. 17: 21-38.

Yu, DL, Shields, DN. (2001). Extension of the parity-space method to fault diagnosis of bilinear systems. Int. J. Syst. Sci. 32: 953-962.

\section{BIOGRAPHIES}

Huanyi Shui received her B.S in Electrical and Computer Engineering at the Shanghai Jiao Tong University, Shanghai, China, in 2012, her M.S in both Mechanical Engineering and Industrial Operation Engineering, and $\mathrm{PhD}$ in Mechanical Engineering at the University of Michigan, Ann Arbor, Michigan USA in 2014, 2017 and 2018 respectively. Her research interests include: fault diagnostics and prognostics in engineering systems, quality control and improvement, signal processing and artificial intelligent techniques in engineering applications.

Shiming Duan received his M.S and PhD in Mechanical Engineering at the University of Michigan, Ann Arbor, in 2007 and 2011 respectively. $\mathrm{He}$ is currently a senior researcher at General Motors Global R\&D Center in Warren, Michigan. His research interests include fault diagnostics and prognostics in automotive systems, modeling and control of dynamic systems, signal processing and machine learning.

Chaitanya Sankavaram is a Senior Researcher with the Vehicle Systems Research Lab at General Motors Global R\&D Center in Warren, Michigan. She joined General Motors in 2013 and since then she has been involved in the research and development of prognostics and health management algorithms for automotive systems. She received her M.S. and Ph.D. degrees in Electrical and Computer Engineering from University of Connecticut, Storrs in 2013 and 2015 respectively. Her research interests include fault diagnostics and prognostics, machine learning, data mining, pattern recognition, reliability analysis, and optimization theory. She was a co-recipient of Walter E. Peterson Best New Technology paper award from IEEE Autotestcon in 2011. She also received the Connecticut Women of Innovation finalist award from the Connecticut Technology Council in 2012. She has published over 25 peerreviewed technical publications and is a member of IEEE, Sigma Xi, and Society of Women Engineers.

Jun Ni received his B.S. degree in Mechanical Engineering from Shanghai Jiao Tong University, Shanghai, China, in 1982, and the M.S. degree and Ph.D. degrees in Mechanical Engineering from the University of Wisconsin-Madison, Madison, USA in 1984 and 1987, respectively. He is currently a Professor of Mechanical Engineering at the University of Michigan, and the director of the S. M. Wu Manufacturing Research Center at the University of Michigan. His research interests include optimization of manufacturing operations, manufacturing process modeling, analysis and design, precision engineering and metrology, intelligent maintenance of industrial systems, quality control, process monitoring, fault diagnostics, and prognostics. 